\title{
Satisfação dos usuários em condições crônicas no âmbito da atenção secundária em saúde
}

Satisfaction of users in chronic conditions within the scope of secondary health care

Gabrielle Borges da Silva ORCID: https://orcid.org/0000-0001-9520-2825 Hospital Naval Marcílio Dias da Marinha do Brasil, Brasil

E-mail: byelle.gbs@gmail.com

Alessandra Sant'anna Nunes

ORCID: https://orcid.org/0000-0001-7435-2568

Universidade do Estado do Rio de Janeiro, Brasil

E-mail: asantnunes@gmail.com

Helena Ferraz Gomes

ORCID: https://orcid.org/0000-0001-6089-6361

Universidade do Estado do Rio de Janeiro, Brasil

E-mail: helenafg1@yahoo.com.br

Carla Tatiana Garcia Barreto Ferrão

ORCID: https://orcid.org/0000-0002-2976-8135

Universidade do Estado do Rio de Janeiro, Brasil

E-mail: carlatgbarreto@gmail.com

Ellen Marcia Peres

ORCID: https://orcid.org/0000-0003-4262-6987

Universidade do Estado do Rio de Janeiro, Brasil

E-mail: ellenperes@globo.com

Bruna Maiara Ferreira Barreto Pires

ORCID: https://orcid.org/0000-0002-5584-8194

Universidade Federal Fluminense, Brasil

E-mail: bruna.barreto07@gmail.com

Priscila Cristina da Silva Thiengo de Andrade

ORCID: https://orcid.org/0000-0003-0840-4838

Universidade do Estado do Rio de Janeiro, Brasil E-mail: profprithiengo@gmail.com

Carolina Cabral Pereira da Costa

ORCID: https://orcid.org/0000-0002-0365-7580 Universidade do Estado do Rio de Janeiro, Brasil E-mail: carolcuerj@hotmail.com

Patrícia Ferraccioli Siqueira Lemos ORCID: https://orcid.org/0000-0002-1345-0612 Universidade do Estado do Rio de Janeiro, Brasil

E-mail: ferraccioli@gmail.com

Thaísa Goulart Lambranho de Azevedo ORCID: https://orcid.org/0000-0001-9418-897X Universidade do Estado do Rio de Janeiro, Brasil E-mail: thaisaazevedo19@gmail.com

Nívia Mariana de Souza Nunes ORCID: https://orcid.org/0000-0002-9207-3826 Universidade do Estado do Rio de Janeiro, Brasil

E-mail: n.mariana.nunes@bol.com.br

Beatriz Camargo Horsts Pereira ORCID: https://orcid.org/0000-0003-0903-0452 Universidade do Estado do Rio de Janeiro, Brasil

E-mail: beatrizhorsts@gmail.com

Daniele Pinto Freire

ORCID: https://orcid.org/0000-0001-7895-8663 Universidade do Estado do Rio de Janeiro, Brasil

E-mail: danielepintofreire@gmail.com

Vivian Sarah Fernandes Vianna Santana

ORCID: https://orcid.org/0000-0001-5153-9549

Universidade do Estado do Rio de Janeiro, Brasil E-mail: viviansarahfvs@gmail.com 
Tainá Ferreira Santos Vilhegas ORCID: https://orcid.org/0000-0003-2863-426X Universidade do Estado do Rio de Janeiro, Brasil E-mail: vilhegastaina@gmail.com

\begin{abstract}
Resumo
Objetivo: avaliar o grau de satisfação de usuários em condições crônicas que fazem uso da terapia biológica em um serviço de atenção secundária em saúde. Método: Pesquisa quantitativa, descritiva e exploratória, realizada em uma Policlínica do Estado do Rio de Janeiro, nos meses de novembro e dezembro de 2017, com 70 usuários portadores de condições crônicas acompanhados nas especialidades de reumatologia, gastroenterologia, alergia e pneumologia que fazem uso da terapia biológica. Utilizou-se um questionário com variáveis referentes as características dos usuários e a satisfação em relação ao serviço. Resultados: Os usuários avaliaram o serviço como satisfatório nas dimensões recepção, atendimento da equipe de enfermagem e saúde, bem como apontaram sentimentos de segurança e confiança na equipe durante o atendimento. Conclusão: a avaliação se mostrou positiva nas diferentes dimensões o que possibilita a tomada de decisão, a partir do conhecimento da performance do serviço sob a visão do usuário. Um excelente grau de satisfação dos usuários promove motivação dos profissionais que os assistem e permitem ao gestor criar estratégias para a continuidade e melhorias nos processos voltados ao acolhimento e assistência destes sujeitos.

Palavras-chave: Doença crônica; Satisfação do paciente; Qualidade da assistência à saúde.
\end{abstract}

\begin{abstract}
Objective: to evaluate the degree of satisfaction of users in chronic conditions who use biological therapy in a secondary health care service. Method: Quantitative, descriptive and exploratory research, carried out in a Polyclinic of the State of Rio de Janeiro, in the months of November and December 2017, with 70 users with chronic conditions monitored in the specialties of rheumatology, gastroenterology, allergy and pneumology that make use of biological therapy. A questionnaire with variables related to the characteristics of users and satisfaction with the service was used. Results: Users evaluated the service as satisfactory in terms of reception, care from the nursing and health team, as well as feeling feelings of security and trust in the team during the service. Conclusion: the evaluation proved to be positive in the different dimensions, which enables decision making, based on the knowledge of the service's performance under the user's view. An excellent degree of user satisfaction promotes motivation of the professionals who assist them and allows the manager to create strategies for continuity and improvements in the processes aimed at welcoming and assisting these subjects.
\end{abstract}

Keywords: Chronic disease; Patient satisfaction; Quality of health care.

\title{
Resumen
}

Objetivo: evaluar el grado de satisfacción de los usuarios en condiciones crónicas que utilizan terapia biológica en un servicio secundario de salud. Método: Investigación cuantitativa, descriptiva y exploratoria, realizada en un Policlínico del Estado de Río de Janeiro, en los meses de noviembre y diciembre de 2017, con 70 usuarios con afecciones crónicas acompañados en las especialidades de reumatología, gastroenterología, alergia y neumología que hacer uso de la terapia biológica. Se utilizó un cuestionario con variables relacionadas con las características de los usuarios y la satisfacción con el servicio. Resultados: Los usuarios evaluaron el servicio como satisfactorio en cuanto a la recepción, atención por parte del equipo de enfermería y salud, así como sentimientos de seguridad y confianza en el equipo durante el servicio. Conclusión: la evaluación resultó positiva en las diferentes dimensiones, lo que permite la toma de decisiones, a partir del conocimiento del desempeño del servicio bajo la mirada del usuario. Un excelente grado de satisfacción de los usuarios promueve la motivación de los profesionales que los atienden y permite al gerente crear estrategias de continuidad y mejora en los procesos encaminados a acoger y atender a estos sujetos.

Palabras clave: Enfermedad crónica; Satisfacción del paciente; Calidad de la atención de salud.

\section{Introdução}

A Organização Mundial da Saúde define o termo "condições crônicas" como todos os problemas de saúde que persistem ao longo do tempo e necessitam de atenção dos serviços de saúde, compreendendo as doenças não transmissíveis, doenças transmissíveis, distúrbios mentais de longo prazo e deficiência física/estrutural permanente (Organização Mundial da Saúde, 2003).

No entanto, as doenças crônicas não transmissíveis (DCNT) são as principais causas de mortes no mundo, além de provocarem um aumento no número de mortes prematuras, perda da qualidade de vida, com alto grau de limitação nas atividades de trabalho e lazer, e gerar gastos econômicos para as famílias, comunidades e a sociedade em geral (Schmidt et. al., 
2011, Organização Mundial de Saúde, 2003). Tais gastos ocorrem tanto de forma direta, decorrentes das internações, medicamentos e tratamentos ambulatoriais, quanto de forma indireta, através da perda de trabalho devido a doença e aposentadorias precoces (Brasil, 2011).

Em relação aos óbitos causados pelas DCNT, mais de 40\% (16 milhões) são mortes em pessoas com menos de 70 anos (Brasil, 2008). No Brasil, as mortes por DCNT correspondem a 72\% dos óbitos, porcentagem proporcionalmente maior que a média mundial. Dentre as principais enfermidades que atingem os brasileiros, destacam-se: o diabetes mellitus, a hipertensão arterial sistêmica, os problemas crônicos de coluna e a dislipidemia (Brasil, 2008; World Health Organization, 2014).

Soma-se a essa estatística, os dados do Ministério da Saúde em parceria com o Instituto Brasileiro de Geografia e Estatística (IBGE), em que 57,4 milhões de pessoas possuem ao menos uma doença crônica não transmissível, correspondendo a aproximadamente $40 \%$ de toda a população brasileira. Este estudo evidencia que essas condições acometem mais o sexo feminino, sendo 34,4 (44,5\%) milhões de mulheres e 23 (33,4\%) milhões de homens (World Health Organization, 2014).

Diante deste panorama o Ministério da Saúde lançou as diretrizes e recomendações para o cuidado integral de DCNT com objetivo de promover reflexão sobre o modelo de assistência responsável em atender as necessidades das pessoas com DCNT, tendo em vista que constituem um sério problema de saúde pública, sendo considerado como um dos focos nacionais e prioritários de atuação (Brasil, 2008; Brasil, 2011).

Além disso, melhorar a saúde de sujeitos e coletividades deve ser considerado investimento no desenvolvimento do país e não apenas um gasto. O ideal é existir um sistema de saúde resolutivo, com articulação de promoção da saúde, vigilância, prevenção e assistência e que, consequentemente, consiga responder efetivamente as necessidades de saúde do país. De acordo com a experiência de outros países, quanto maior as ações de promoção da saúde e de prevenção de DCNT e seus fatores de risco, de maneira integrada e abrangente, maior será a redução dos fatores de risco e da prevalência das DCNT (Brasil, 2008; Brasil, 2011).

Neste contexto, é primordial a organização dos serviços, e a Rede de Atenção à Saúde deve buscar fazê-lo através de arranjos delineados conforme as densidades tecnológicas, sendo elas: a Atenção Primária à saúde (APS), a Atenção Secundária à saúde e a Atenção terciária à saúde, sendo igualmente importantes, se diferenciando apenas pelas diferentes densidades tecnológicas que as caracterizam (Brasil, 2010).

No entanto, apenas os serviços de APS não são suficientes para atender às necessidades em saúde da população. Por isso, os serviços da APS devem ser apoiados e complementados pela Atenção Secundária, para a realização de ações e cuidados especializados (Brasil, 2010).

A atenção secundária em saúde é estratégica, pois articula os diferentes níveis de atenção e desempenha um papel de ampliação do acesso às consultas e procedimentos especializados, sendo imprescindível na resolubilidade e integralidade do cuidado. A boa qualidade de atendimento na atenção secundária resulta em resolutividade na atenção primária, gerando assim a integralidade da assistência (Brasil, 2010; Erdmann et al., 2013). Além disso, a resolutividade nos diferentes níveis de atenção à saúde permite a longitudinalidade do cuidado.

Dentre os diversos profissionais que atuam na atenção secundária, tem-se os profissionais de enfermagem, em especial o enfermeiro, que desempenha diversas atividades, como: promoção da saúde, prevenção de doenças e agravos, tratamento, reabilitação e atividades de cuidado.

Em relação as atividades de cuidado, destaca-se: o acolhimento, a coleta de materiais para exames, o atendimento em casos de acidentes de trabalho, a consulta de enfermagem, além de organizarem reuniões de equipe, a triagem dos usuários, desenvolver o atendimento multiprofissional, a visita domiciliar e demais atividades gerenciais. Quanto à medicação, os enfermeiros podem ser os responsáveis pela dispensação, solicitação, supervisão, administração, controle da dispensação e das 
receitas. Cabe ainda ao profissional investir na capacitação profissional através da educação continuada e permanente, e no desenvolvimento de pesquisas científicas baseadas em evidências (Ferreira, 2012).

Ressalta-se que uma das formas de realizar a manutenção da qualidade de atendimento é através da avaliação, a partir da perspectiva do cliente sobre o serviço. A opinião do usuário poderá ser utilizada como base para o planejamento e a tomada de decisões, podendo modificar as deliberações dos prestadores e dos organizadores do serviço. Usuários satisfeitos aderem melhor ao tratamento, gerando resultado positivo para a sua saúde (Paiva et al., 2015).

Contudo, torna-se necessário que os serviços prestados ao usuário sejam efetivos e de qualidade, sendo fundamental a avaliação do grau de satisfação, pois trata-se de um bom indicador sobre a qualidade do serviço prestado, principalmente do serviço de enfermagem, por ser o profissional que participa efetivamente de ações de promoção da saúde, prevenção de agravos e na prestação dos cuidados nos diferentes níveis de atenção à saúde (Paiva et. al., 2015).

Diante do exposto, estabeleceu-se o seguinte problema de pesquisa: Qual o grau de satisfação dos usuários em condições crônicas que fazem uso da terapia biológica atendidos em um serviço de atenção secundária em saúde? Definiu-se como hipótese que o grau de satisfação dos usuários é positivo, variando entre bom e muito bom, conforme a experiência e vivência direta durante as infusões da terapia e as consultas com os profissionais.

Logo, tem-se por objetivo: avaliar o grau satisfação de usuários em condições crônicas que fazem uso da terapia biológica em um serviço de atenção secundária em saúde.

\section{Metodologia}

Pesquisa quantitativa, descritiva e exploratória. A pesquisa quantitativa se baseia na objetividade e considera que a compreensão da realidade se dá apenas através da análise de dados, com auxílio de instrumentos padronizadas e neutros (Polit, Beck \& Hungler, 2004; Pereira et al., 2018).

O campo foi uma unidade de terapia biológica de um serviço de atenção secundária em saúde numa Policlínica no Estado do Rio de Janeiro. A unidade é inserida no nível de atenção secundária em saúde e oferece atendimento ambulatorial em 27 especialidades. É dotada de um centro cirúrgico ambulatorial para cirurgias-dia de pequeno e médio porte, laboratórios acadêmicos, atendimento diagnóstico e terapêutico, programas e projetos com foco para a medicina preventiva. A unidade de terapia biológica funciona dentro de um setor que presta atendimento de emergência e urgência a pacientes que, ao comparecerem para uma consulta ambulatorial ou realização de exame diagnóstico, necessitem de atendimento.

A população do estudo correspondeu a 80 usuários portadores de condições crônicas acompanhados nas especialidades de reumatologia, gastroenterologia, alergia e pneumologia que fazem uso da terapia biológica. A amostra foi selecionada por meio de amostragem probabilística por conveniência, a partir dos seguintes critérios de inclusão: idade a partir de 18 anos; diagnóstico médico de alguma doença gástrica, reumatológica, pulmonar ou alérgica que justifique o uso da terapia biológica; estar em tratamento na referida unidade por no mínimo 6 meses. E por critérios de exclusão: menores de 18 anos, que durante esse período da infância/ adolescência passam por diversas transformações biológicas, psicológicas e comportamentais.

Destaca-se que tais critérios de exclusão, baseiam-se no fato de que as mudanças decorrentes da terapêutica são difíceis para o jovem com doença crônica, face as próprias transformações inerentes a faixa etária (Araújo et al., 2011). Tais contextos emocionais e psíquicos poderiam influenciar na opinião desses sujeitos, gerando inexatidões no resultado do estudo.

No entanto, obteve-se uma amostra final de 70 usuários portadores de condições crônicas que fazem uso de terapia biológica, gerando um poder amostral maior que $80 \%$. Os usuários responderam um questionário padronizado, após o atendimento no serviço de terapia biológica, conforme agendamento prévio das infusões da terapia e das consultas com os profissionais. 
O questionário era composto por 12 perguntas fechadas, com as seguintes variáveis preditoras: sexo, idade, escolaridade, especialidade de atendimento de origem, terapia biológica utilizada, diagnóstico médico principal, atendimento da recepção, atendimento da equipe de enfermagem e saúde, segurança/confiança na equipe de saúde, informações e esclarecimentos sobre o seu próprio estado de saúde e variável desfecho: grau de satisfação do usuário.

Os dados foram organizados, digitados e analisados no programa Epi-Info versão 7.2.2.2, utilizando a estatística simples, por meio de frequências absoluta e relativa.

Foram respeitados todos os aspectos éticos, conforme Resolução no 466 de 2012 do Conselho Nacional de Saúde, e aprovada no Comitê de Ética em Pesquisa sob o número de parecer 2.250.579 (Brasil, 2012).

\section{Resultados}

Quanto a caracterização dos usuários do serviço de terapia biológica, 68,75\% (n=55) são do sexo feminino, 62,50\% $(\mathrm{n}=50)$ apresentam idade superior a 40 anos e, $40 \%(\mathrm{n}=28)$ possuem o ensino médio completo.

Dentre as especialidades de atendimento de origem, em relação ao perfil clínico dos usuários, 54\% (n=43) são oriundos do ambulatório de Gastroenterologia.

Em relação a terapia biológica utilizada, há prevalência do Infliximabe, utilizada por 55\% (n=44) dos usuários e 60\% $(\mathrm{n}=48)$ fazem tratamento há mais de 24 meses. Os diagnósticos médicos mais frequentes são: doença de Chron, Retocolite ulcerativa e Artrite reumatóide.

No que se refere a avaliação da satisfação dos usuários do serviço foram avaliadas o atendimento da recepção, bem como o atendimento por parte da equipe de enfermagem e saúde, a segurança e confiança na equipe, informações e esclarecimentos do atendimento e, a própria unidade de uma forma geral, conforme apresentado na (Tabela 1).

Tabela 1: Distribuição da avaliação de satisfação do usuário em um serviço de terapia biológica de uma Policlínica em relação ao atendimento ofertado. Rio de Janeiro, Brasil, 2020.

$\begin{array}{lcc}\text { Variáveis } & \text { N } & \text { \% } \\ \text { Como o(a) senhor(a) avalia o atendimento da recepção? } & & \\ \text { Muito Bom } & 56 & 80,00 \\ \text { Bom } & 11 & 15,71 \\ \text { Regular } & 02 & 02,86 \\ \text { Ruim } & - & - \\ \text { Muito ruim } & - & - \\ \text { Não informou } & 01 & 01,43\end{array}$

Como o(a) senhor(a) avalia o atendimento da equipe de enfermagem/saúde?

$\begin{array}{lcc}\text { Muito Bom } & 58 & 82,86 \\ \text { Bom } & 10 & 14,28 \\ \text { Regular } & & - \\ \text { Ruim } & 01 & 01,43 \\ \text { Muito ruim } & 01 & 01,43\end{array}$

Não informou

$\mathrm{O}$ (a) senhor(a) sentiu segurança/confiança na equipe de saúde durante o atendimento?

Sim

67

95,72

Não

Não informou 
Como o (a) senhor(a) avalia as informações e esclarecimentos que você teve sobre o seu estado de saúde no estabelecimento?

$\begin{array}{lcc}\text { Muito Bom } & 34 & 48,57 \\ \text { Bom } & 31 & 44,29 \\ \text { Regular } & 03 & 04,28 \\ \text { Ruim } & - & - \\ \text { Muito ruim } & 01 & 01,43 \\ \text { Não informou } & 01 & 01,43\end{array}$

De uma maneira geral, como o(a) senhor(a) avalia este estabelecimento de saúde?

$\begin{array}{lcc}\text { Muito Bom } & 23 & 32,86 \\ \text { Bom } & 33 & 47,14 \\ \text { Regular } & 11 & 15,71 \\ \text { Ruim } & - & - \\ \text { Muito ruim } & - & - \\ \text { Não informou } & 03 & 04,29\end{array}$

$\begin{array}{lll}\text { Total } & 70 & 100\end{array}$

Fonte: Autores (2020).

Observa-se na Tabela 1 que $80 \%(n=56)$ consideram o atendimento da recepção muito bom, e 82,86 (n=58) consideram o atendimento da equipe de enfermagem/saúde muito bom. Quanto a segurança/confiança da equipe de saúde durante o atendimento $95,72 \%(n=67)$ consideram muito bom. No entanto, em relação as informações recebidas sobre o estado de saúde, observa-se que os usuários consideraram muito bom em 48,57\% ( $\mathrm{n}=32$ ) e bom em 44,29\% ( $\mathrm{n}=31$ ). De modo geral, o estabelecimento de saúde foi avaliado como muito bom e bom, respectivamente.

\section{Discussão}

Ao analisar o perfil dos usuários atendidos no serviço de terapia biológica observa-se a predominância do sexo feminino, tais dados corroboram com estudo realizado em um serviço ambulatorial de um Hospital Universitário em que 75,5\% eram mulheres (Marques et al., 2016). Contudo, não foi possível correlacionar no presente estudo o grau de satisfação dos usuários com relação ao sexo, idade e escolaridade no presente estudo. No entanto, infere-se que as mulheres procuram mais os serviços de saúde e tendem a uma maior satisfação.

Estudo sobre a utilização de serviços de saúde por usuários com doenças crônicas não transmissíveis, à partir da Pesquisa Nacional de Saúde no Brasil, evidenciou que as mulheres utilizam mais os serviços de saúde, tanto em relação as consultas quanto para internações (Malta et al., 2017).

Ademais, estudo realizado em um município de Pernambuco sobre a satisfação de usuários com os serviços ofertados pelo SUS desvelou que o sexo feminino e a baixa escolaridade foram características independentemente associadas ao maior uso e satisfação por parte dos usuários (Cruz et al., 2017).

Em relação as dimensões da avaliação do grau de satisfação dos usuários, estudo aponta que o acolhimento engloba: 1- o contato inicial do usuário com o serviço, através da recepção e 2- o trabalho do profissional em serviço. Por isso, o primeiro contato deve ser avaliado, a fim de garantir uma boa qualidade de acesso ao serviço desde o início. O estudo evidenciou que 95,71\% dos usuários consideram o atendimento da recepção "muito bom” ou "bom", demonstrando que o contato inicial com o serviço, através da recepção, se mostra satisfatório (Ayach, 2015). 
Quanto a satisfação com o atendimento profissional, estudo identificou à satisfação, percebida por usuários, com o cuidado recebido da equipe de saúde, classificados por 76,7\% como satisfeitos e 23,3\% insatisfeitos (Protasio et al., 2017). No entanto, os dados do presente estudo apresentam valores de satisfação maiores, em que 97,14\% dos usuários encontram-se satisfeitos com o atendimento da equipe de enfermagem e saúde

Além disso, são considerados essenciais para os usuários o apoio afetivo do profissional, as informações de saúde que lhe são passadas, sua participação na tomada de decisão sobre o seu próprio tratamento, condições fundamentais para a solidificação do sentimento de segurança nas competências técnicas e profissionais (Ferreira et al., 2016)

Nesta perspectiva, o estudo aponta que 95,72\% dos usuários sentem segurança e confiança na equipe de saúde durante o atendimento. O sentimento de segurança e confiança acima de 80\% foi constatado em outros estudos com usuários (Ayach, 2015; Brandão et al., 2015). Infere-se que a confiança e segurança em relação ao profissional que os assiste é fundamental para a criação de vínculo e continuidade do tratamento.

A atuação multidisciplinar, na atenção as pessoas com doenças crônicas, é fundamental, pois o sucesso do tratamento depende da participação ativa e envolvimento do usuário (Brasil, 2013).

No tratamento de pacientes crônicos, a parceria entre profissionais e usuários garante, ao considerar o ponto de vista do paciente, maior satisfação, melhora da adesão e uma maior continuidade dos cuidados (Holman \& Lorig, 2000).

Quanto as informações e esclarecimentos que recebem sobre o seu estado de saúde observa-se que 92,86\% dos usuários consideram "muito bom" ou "bom".

Os esclarecimentos e informações repassadas pela equipe de saúde são fundamentais para o manejo do tratamento, permitindo a prevenção de complicações, a promoção da saúde e a qualidade de vida do paciente com DCNT.

A prevenção e o controle das doenças crônicas exigem modificações relacionados ao estilo de vida e hábitos alimentares, bem como atividade físicas, adesão ao tratamento, entre outros, medidas que requerem a ações efetivas de educação em saúde dos diversos profissionais, nas diferentes áreas de atuação, a nível individual e coletivo (Azevedo et al., 2018). Daí a importância das informações e esclarecimentos repassados pela equipe de saúde.

$\mathrm{Na}$ avaliação do estabelecimento de saúde, $80 \%$ dos usuários se dizem satisfeitos com o serviço ofertado, dado relevante na perspectiva da gestão em saúde, e conforme aponta estudo a satisfação do usuário é uma diretriz para a orientação da gestão em saúde na implementação de melhorias nos serviços públicos (Cruz et al., 2017). Ainda, usuários satisfeitos aderem melhor ao tratamento, e uma boa adesão é fundamental para melhor resultado no contexto de saúde (Ferreira, 2008).

Dentre as limitações do estudo destaca-se que não houve associações da satisfação com relação ao sexo, escolaridade, idade e tempo de tratamento no serviço, o que permitiria um maior aprofundamento na discussão dos resultados. Além disso, existem poucos estudos de avaliação do grau de satisfação dos usuários nos serviços de saúde, principalmente a nível ambulatorial o que limita a discussão dos resultados.

Ressalta-se, ainda, que a coleta de dados ocorreu num período de extrema dificuldade econômica da unidade, frente a crise que se abateu no Estado do Rio de Janeiro.

Contudo, reforça-se a importância de estudos que avaliam a satisfação do usuário, a fim de subsidiar melhorias no processo de trabalho e permitir uma gestão efetiva do trabalho em enfermagem e saúde.

\section{Conclusão}

O estudo possibilitou caracterizar os usuários do serviço de terapia biológica de uma Policlínica no Estado do Rio de Janeiro em relação ao sexo, idade, escolaridade, terapia biológica mais utilizada e os principais diagnósticos médicos, viabilizando o direcionamento das ações e a avaliação do grau de satisfação em relação ao serviço.

A avaliação da satisfação se mostrou positiva nas diferentes dimensões avaliadas o que possibilita a tomada de 
decisão, a partir do conhecimento da performance do serviço sob a visão do usuário.

Ainda, mesmo diante das adversidades o serviço se apresentou satisfatório para os usuários, o que demonstra a importância do acolhimento, comunicação e direcionamento no contexto de cuidado aos usuários com DCNT por parte dos profissionais de saúde.

Além disso, um excelente grau de satisfação dos usuários promove motivação dos profissionais que os assistem e permitem ao gestor criar estratégias para a continuidade e melhorias nos processos voltados ao acolhimento e assistência.

Sugere-se a realização de outros estudos com maior número de usuários voltados para avaliação da satisfação dos serviços à nível ambulatorial.

\section{Referências}

Araújo, Y. B. de, Neusa, C, Gomes, I. P, \& Nóbrega, R. D da. (2011). Enfrentamento do adolescente em condição crônica: importância da rede social. Rev. bras. enferm. 1 64(2): 281-286. http://dx.doi.org/10.1590/S0034-71672011000200010.

Ayach, C. (2015). A visão do usuário sobre o serviço público odontológico e a autopercepção em saúde bucal. $121 \mathrm{f}$. Tese (doutorado) - Universidade Estadual Paulista Júlio de Mesquita Filho, Faculdade de Odontologia de Araçatuba, 2015.

Azevedo, P. R. A., Sousa, M. M., Sousa, N. F., \& Oliveira, S. H. dos S. (2018). Ações de educação em saúde no contexto das doenças crônicas: revisão integrativa. Rev Fund Care Online. 10(1):260-267. http://dx.doi.org/10.9789/2175-5361.rpcfo.v10.5013.

Brandão, O. S., Pierantoni, C. R., Gouvea, C. S. D. \& França, T. (2015). O desempenho da maternidade da Santa Casa de Angra dos Reis face aos critérios de qualidade do Programa Nacional de Avaliação dos Serviços de Saúde. In: Pierantoni, C. R., Viana, C.M., Parada, R. (Org.). Gestão de Sistemas de Saúde no Rio de Janeiro. 1ed.Rio de Janeiro: CEPESC, 1, 161-192. Brasil.

Brasil. Ministério da Saúde (2008). Secretaria de Vigilância à Saúde. Secretaria de Atenção à Saúde. Diretrizes e recomendações para o cuidado integral de doenças crônicas não-transmissíveis: promoção da saúde, vigilância, prevenção e assistência. Brasília: Ministério da Saúde.

Brasil. Ministério da Saúde (2010). Portaria $n^{\circ}$ 4.279, de 30 de dezembro de 2010. Diário Oficial da União, Brasília-DF, Seção 1, p. 89, 31 dez. 2010. Estabelece diretrizes para a organização da Rede de Atenção à Saúde no âmbito do Sistema Único de Saúde (SUS). Diário Oficial da União, Poder Executivo, Brasília, DF, 31 dez. 2010, Seção 1.

Brasil. Ministério da Saúde (2011). Secretaria de Vigilância em Saúde. Departamento de Análise de Situação de Saúde. Plano de ações estratégicas para o enfrentamento das doenças crônicas não transmissíveis (DCNT) no Brasil 2011-2022. Brasília: Ministério da Saúde.

Brasil. Ministério da Saúde (2012). Conselho Nacional de Saúde. Resolução n. 466, de 12 de dezembro de 2012. Aprova diretrizes e normas regulamentadoras de pesquisas envolvendo seres humanos. Brasília, Diário Oficial da União, 12 dez. 2012.

Brasil. Ministério da Saúde (2013). Diretrizes para o cuidado das pessoas com doenças crônicas nas redes de atenção à saúde e nas linhas de cuidado prioritárias. Brasília-DF, 2013.

Cruz, I. L. C. M., Parente, A. S., Mesquita, F. O. S., \& Jerônimo, A. S. (2017). Satisfação dos usuários com os serviços do Sistema Único de Saúde - SUS em um município do sertão de Pernambuco. Id online Rev. Mult. Psic. 11(39).

Erdmann, A. L., Andrade, S. R. de., Mello, A. L. S. F de. \& Drago, L. C. (2013). A atenção secundária em saúde: melhores práticas na rede de serviços. Rev. Latino-Am. Enfermagem. 21(spe): 131-139. http://dx.doi.org/10.1590/S0104-11692013000700017.

Ferreira, G. M. M. (2012). O Processo de trabalho do enfermeiro na atenção secundária do Sistema Único de Saúde, p. 50. Trabalho de Conclusão de Curso apresentado ao Curso de Graduação em Enfermagem do Centro de Ciências da Saúde. Universidade Regional de Blumenau. Santa Catarina.

Ferreira, P. H. C., Guedes, H., Moreira, A. S., Baracho, V. S., Caldeira, A. B. R., Guedes, C. F., Ferreira, T. S., \& Ribeiro, L. C. C. (2016). Satisfação dos clientes externos quanto aos cuidados de enfermagem. Revista Mineira de Enfermagem. Minas Gerais, $20, \mathrm{e} 975$.

Holman H., \& Lorig K. (2000). Patients as partners in managing chronic disease. Partnership is a prerequisite for effective and efficient health care. BMJ. 320(7234):526-7. https://doi.org/10.1136/bmj.320.7234.526.

Malta, D. C., Bernal, R. T. I., Lima, M. G., Araújo, S. S. C. de., Silva, M. M. A. da., Freitas, M. I. F., \& Barros, M. B. A. (2017). Doenças crônicas não transmissíveis e a utilização de serviços de saúde: análise da Pesquisa Nacional de Saúde no Brasil. Revista de Saúde Pública, 51(1), 4s. https://doi.org/10.1590/s1518-8787.2017051000090

Marques, I. P., Andrade, V. L., Oliveira Júnior, L. B. de., Costa, F. M. da. (2016). Pesquisa de satisfação do usuário do SUS realizada no Hospital Universitário da UFJF. HU Revista, Juiz de Fora. 42(1),.67-74.

Organização Mundial da Saúde (2003). Cuidados inovadores para condições crônicas: componentes estruturais de ação: relatório mundial. Brasília: OMS, 2003 .

Paiva, M. B. P. de, Mendes, W., Brandão, A. L., Campos, C. E. A. (2015). Uma contribuição para a avaliação da Atenção Primária à Saúde pela perspectiva do usuário. Physis; 25(3): 925-950. http://dx.doi.org/10.1590/S0103-73312015000300013. 
Research, Society and Development, v. 10, n. 3, e25010313288, 2021

(CC BY 4.0) | ISSN 2525-3409 | DOI: http://dx.doi.org/10.33448/rsd-v10i3.13288

Pereira, A. S., Shitsuka, D. M., Pereira, F. J. \& Scitsuka, R. (2018). Metodologia da pesquisa científica. UFSM.

Polit, D. F., Beck, C. T. \& Hungler, B. P. (2004). Fundamentos de pesquisa em enfermagem: métodos, avaliação e utilização. (5a ed.), Artmed.

Protasio, A. P. L., Gomes, L. B, Machado, L. S, Valença, A. M. G. (2017) Satisfação do usuário da Atenção Básica em Saúde por regiões do Brasil: $1^{\circ}$ ciclo de avaliação externa do PMAQ-AB. Ciênc. saúde coletiva. 22(6): 1829-1844. https://dx.doi.org/10.1590/1413-81232017226.26472015.

Schmidt, M. I., Duncan, B. B., Azevedo e Silva, G., Menezes, A. M., Monteiro, C. A., Barreto, S. M., Chor, D., \& Menezes, P. R. (2011). Chronic noncommunicable diseases in Brazil: burden and current challenges. Lancet. 4; 377(9781):1949-61. https://doi.org/10.1016/S0140-6736(11)60135-9

World Health Organization. (2014). Global status report on noncommunicable diseases 2014. Switzerland: WHO, 2014. 